\title{
Survival after recurrence in patients with gastric cancer who receive S-1 adjuvant chemotherapy: exploratory analysis of the ACTS-GC trial
}

Seiji Ito ${ }^{1^{*}}$, Yasuo Ohashi ${ }^{2}$ and Mitsuru Sasako ${ }^{3}$

\begin{abstract}
Background: Some patients develop recurrence after curative resection and adjuvant chemotherapy. S-1, an oral fluoropyrimidine, is one of the standard regimens in adjuvant chemotherapy, and is also used in first-line treatment for advanced/metastatic gastric cancer. It is controversial as to whether the same treatment strategy can be applied for patients who develop recurrence after adjuvant chemotherapy and those who did not receive adjuvant chemotherapy. To investigate this issue, we compared the outcomes of patients who developed recurrences after treatment with or without adjuvant chemotherapy using the results of the Adjuvant Chemotherapy Trial of S-1 for Gastric Cancer (ACTS-GC).
\end{abstract}

Methods: Patients who had confirmed recurrence in the ACTS-GC trial were analyzed. We defined 2 independent cohorts. Cohort 1 patients were divided by whether they received adjuvant chemotherapy (adjuvant S-1 group and surgery-only group). Cohort 2 patients were divided by whether they received a regimen including S-1 (IS) or not including S-1 (NIS) after recurrence.

Results: A total of 375 patients experienced recurrence (160 in the adjuvant S-1 group and 215 in the surgery-only group). In cohort 1, the median time from recurrence to death (TFRD) was 11.4 months ( $95 \%$ confidence interval [Cl], 8.4-13.9) in the adjuvant S-1 group and 11.3 months (95\% Cl, 9.7-13.1) in the surgery-only group (hazard ratio [HR], 1. 05; 95\% Cl, 0.84-1.31). In cohort 2, 292 patients received chemotherapy after recurrence and were divided into the IS $(n=189)$ or the NIS group $(n=103)$. The median TFRD was 13.9 months $(95 \% \mathrm{Cl}, 12.7-15.6)$ in the IS group and 8.1 months $(95 \% \mathrm{Cl}, 6.6-9.7)$ in the NIS group ( $\mathrm{HR}, 0.59 ; 95 \% \mathrm{Cl}, 0.45-0.76)$, and there was no significant interaction between the adjuvant S-1 group and surgery-only group.

Conclusion: Adjuvant chemotherapy with S-1 prolonged overall survival without influencing the TFRD. The same treatment strategy may be applied for patients who develop recurrence after adjuvant chemotherapy and those who did not receive adjuvant chemotherapy.

Trial registration: NCT00152217. First Posted on September 9, 2005.

Keywords: ACTS-GC, Adjuvant chemotherapy, S-1, Recurrent gastric cancer, Stomach neoplasms

\footnotetext{
*Correspondence: seito@aichi-cc.jp

'Department of Gastroenterological Surgery, Aichi Cancer Center, 1-1

Kanokoden, Chikusa-ku, Nagoya, Aichi 464-8681, Japan

Full list of author information is available at the end of the article
}

(c) The Author(s). 2018 Open Access This article is distributed under the terms of the Creative Commons Attribution 4.0 International License (http://creativecommons.org/licenses/by/4.0/), which permits unrestricted use, distribution, and reproduction in any medium, provided you give appropriate credit to the original author(s) and the source, provide a link to the Creative Commons license, and indicate if changes were made. The Creative Commons Public Domain Dedication waiver (http://creativecommons.org/publicdomain/zero/1.0/) applies to the data made available in this article, unless otherwise stated. 


\section{Background}

Gastric cancer is the third leading cause of cancerrelated death [1] and there were 723,100 deaths from gastric cancer worldwide in 2012. The mainstay of treatment for gastric cancer is surgery. In addition to surgery, clinical trials have shown that preoperative and/or postoperative adjuvant treatment is beneficial for potentially curable gastric cancer, although different approaches are used in the USA, European Union, and Asia [2-9].

The results of the Adjuvant Chemotherapy Trial of S-1 for Gastric cancer (ACTS-GC), a Japanese trial evaluating the effectiveness of 1 year of postoperative treatment with S-1, showed a significant survival benefit over surgery alone for patients with stage II or III gastric cancer according to the Japanese Classification of Gastric Carcinoma (Second English Edition) [10].

S-1 (Taiho Pharmaceutical, Tokyo, Japan) is an oral fluoropyrimidine that combines tegafur, a prodrug of 5fluorouracil with gimeracil and oteracil potassium in a molar ratio of 1:0.4:1 [11]. The 5-year overall survival (OS) rate, the primary endpoint of the ACTS-GC at 5 years was $71.7 \%$ in the adjuvant $S-1$ group and $61.1 \%$ in the surgery-only group, demonstrating the usefulness of postoperative adjuvant chemotherapy with S-1 (hazard ratio $[\mathrm{HR}], 0.67 ; 95 \%$ confidence interval [CI], 0.54-0.83) $[12,13]$. The relapse-free survival rate at 5 years was 65 . $4 \%$ in the adjuvant S-1 group and $53.1 \%$ in the surgeryonly group (HR, 0.65 ; 95\% CI, 0.54-0.79). On the basis of these results, S-1 is currently used as standard postoperative adjuvant chemotherapy for gastric cancer in Japan and Asian countries. However, approximately $35 \%$ of patients still develop recurrences after curative resection and adjuvant chemotherapy.

In colorectal, ovarian, and breast cancer, the strategy of reuse of the same drug as that used for postoperative adjuvant chemotherapy has been studied in patients who had postoperative recurrences (colorectal cancer: ACCENT [14], MOSAIC [15, 16], and NSABP C-07 [17, 18]; ovarian cancer: Markman et al. [18], ICON4/ AGO-OVAR-2.2 [19], Harries et al. [20] and Fung et al. [21]; and breast cancer: RHEA [22]). In these cancers, similar to gastric cancer, the same drugs are used for adjuvant chemotherapy and for advanced/metastatic cancer treatment. In Asian and some European countries, $\mathrm{S}-1$ is used as adjuvant chemotherapy and also as first-line treatment for advanced/metastatic gastric cancer. It is unclear whether regimens including $\mathrm{S}-1$ show similar efficacy in those who develop recurrence after $\mathrm{S}-1$ adjuvant chemotherapy as those who are naïve to $\mathrm{S}-1$. Thus, we performed this exploratory analysis using the results of the ACTS-GC trial to investigate the duration of survival after recurrence comparing those who previously received a regimen including $\mathrm{S}-1$ with those had not received $\mathrm{S}-1$.

\section{Methods}

Patients, treatments, and follow-up

Patients were randomly assigned to either the adjuvant S-1 group or the surgery-only group in the ACTS-GC. The institutions participated in the ACTS-GC were shown in Additional file 1: Text S1. The main inclusion criteria were as follows: age 20-80 years, histologically confirmed stage II, IIIA, or IIIB after curative surgery, D2 or more extensive lymph node dissection with R0 surgery, adequate oral intake, and preserved major organ function. Tumor stage classification and D classification were in accordance with the Japanese Classification of Gastric Carcinoma (Second English Edition) [10]. The detailed inclusion and exclusion criteria were described previously [12].

Patients assigned to the adjuvant $\mathrm{S}-1$ group received S-1 as adjuvant chemotherapy. S-1 was orally administered at a dose corresponding to the patient's body surface area (BSA) $\left(40 \mathrm{mg}\right.$ with $\mathrm{BSA}<1.25 \mathrm{~m}^{2} ; 50 \mathrm{mg}$ with BSA $1.25-1.5 \mathrm{~m}^{2} ; 60 \mathrm{mg}$ with BSA $>1.5 \mathrm{~m}^{2}$ ) twice daily after meals for 28 consecutive days, followed by a 14-day rest period. Treatment was continued for 1 year after surgery.

Patients assigned to the surgery-only group received no anticancer treatment postoperatively until recurrence was confirmed either clinically or with imaging studies, which included ultrasonography, computed tomography, gastrointestinal radiography, and endoscopy. Every patient was followed up for 5 years after the date of surgery or death. Those who were alive at 5 years were censored at this point. Treatment after recurrence was not specified in the original protocol. Further details have been reported previously [12, 13]. Patients who had confirmed diagnosis of recurrent gastric cancer were included in this analysis.

\section{Cohort definition}

We defined 2 different cohorts. In cohort 1, patients were divided by whether they received adjuvant chemotherapy. This cohort was used to investigate whether there were any differences in survival after recurrence between patients in the $\mathrm{S}-1$ and surgery alone arm.

Cohort 2 patients were divided by whether they received a regimen including S-1 (IS) or not including S1 (NIS) after recurrence. This cohort was used to investigate whether the efficacy of reuse of S-1 after recurrence was the same in those who received S-1 as adjuvant chemotherapy and those who were completely chemotherapy naïve. The IS group consisted of patients who received S-1-based chemotherapy for treatment of recurrent gastric cancer.

Patients assigned to the adjuvant S-1 group were divided into 2 groups according to whether they had 
developed recurrent disease within 6 months after the completion of adjuvant chemotherapy with S-1 (including recurrence during postoperative adjuvant chemotherapy with $S-1$ ), or at 6 or more months after the completion of adjuvant chemotherapy. This classification was based on the fact that recurrent tumors within 6 months after termination of adjuvant chemotherapy showed a different response to chemotherapy regimens in some other cancers, such as ovarian cancer.

\section{Statistical analysis}

Time from recurrence to death (TFRD) was defined as the interval from the date of recurrence to the date of death from any cause. Survival rates were estimated by the Kaplan-Meier method. A Cox proportional hazards model was used to calculate HRs. A subgroup analysis was performed for the factors that may influence the outcome. Multivariate survival analysis was performed using a Cox proportional hazards model after checking for validity of the proportional hazards assumption by plotting log-minus-log survival curves. The distributions of patient characteristics between the two groups were compared using chisquare test. Results were defined as statistically significant at $P<0.05$. All statistical analyses were performed with SAS software version 9.1 (SAS Institute Inc., Cary, NC, USA).

\section{Results}

\section{Patients}

Within 5 years after surgery, 375 out of 1034 eligible patients experienced recurrence (160 in the adjuvant S-1 group and 215 in the surgery-only group). Among these patients, $121(75.6 \%)$ in the adjuvant S-1 group and 171 $(79.5 \%)$ in the surgery-only group received chemotherapy after recurrence (Fig. 1). Eighty-three out of 375 received non-chemotherapeutic treatment (e.g., surgery, radiotherapy, or best supportive care: 39 in the adjuvant $\mathrm{S}-1$ group and 44 in the surgery-only group). To treat the recurrence, $\mathrm{S}$-1-based regimens were administered to 57 of $121(35.6 \%)$ in the adjuvant S-1 group and 132 of $171(61.4 \%)$ in the surgery-only group $(P<0.001)$ (Table 1).

\section{TFRD (adjuvant S-1 group and surgery-only group)}

The TFRD was analyzed according to the treatment group assigned at the time of enrollment in the ACTSGC (adjuvant S-1 or surgery-only group) (Fig. 2a). The median duration of follow-up from recurrence was 43. 4 months. The median TFRD was 11.4 (95\% CI, 8.4-13.9) and 11.3 months in the adjuvant S-1 group $(n=160)$ and the surgery-only group $(n=215)$, respectively. The HR for death in the adjuvant S-1 group, as compared with the surgery-only group, was 1.05 (95\% CI, 0.84-1.31). Among patients who received chemotherapy after the recurrence (adjuvant S-1

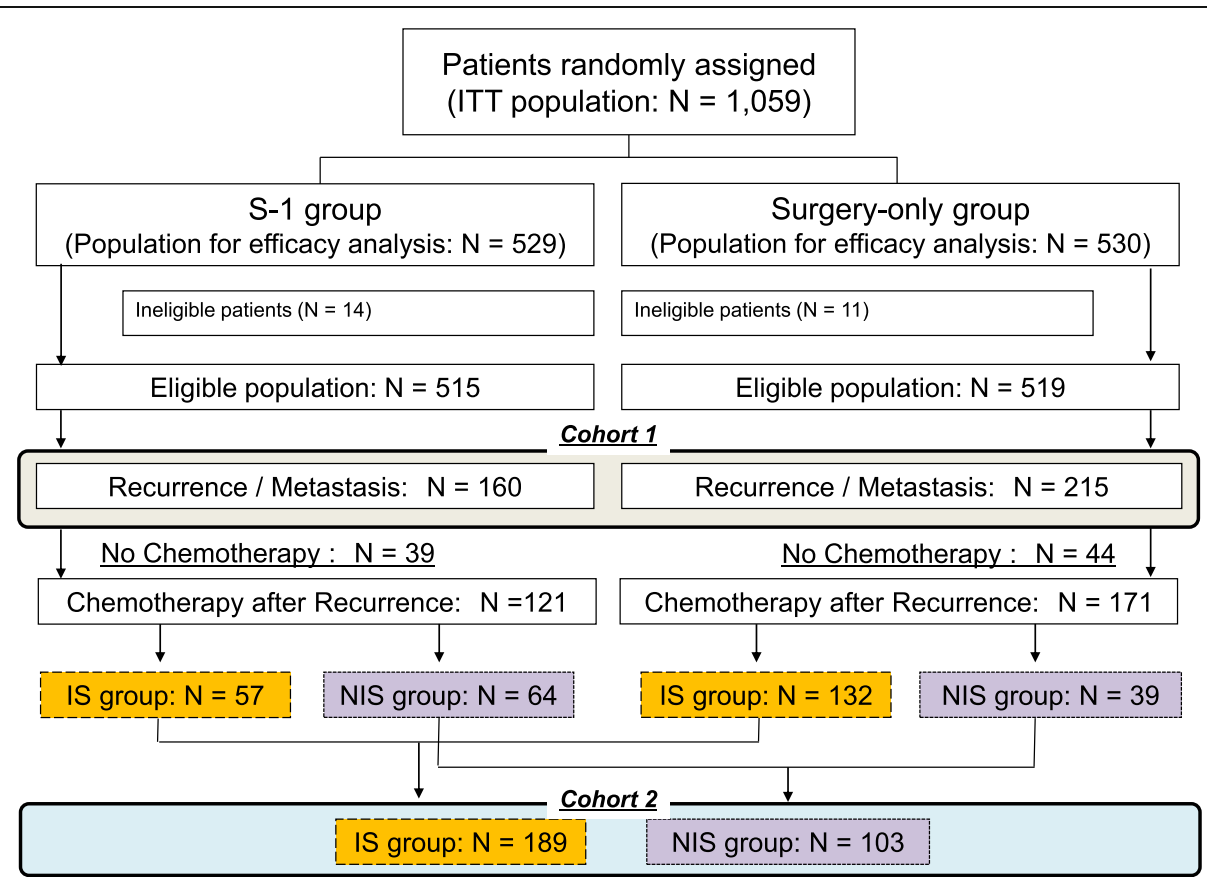

Fig. 1 CONSORT diagram. Among eligible patients enrolled in the ACTS-GC, patients confirmed to have recurrence were included in this analysis. D1, D1 lymphadenectomy; ITT, intention to treat 
Table 1 Patient characteristics in cohort 1

\begin{tabular}{|c|c|c|c|c|}
\hline Cohort 1 & & $\begin{array}{l}\text { S-1 adjuvant } \\
(n=160)\end{array}$ & $\begin{array}{l}\text { Surgery only } \\
(n=215)\end{array}$ & $\begin{array}{l}X \text { square test } \\
p \text {-value }\end{array}$ \\
\hline \multirow[t]{2}{*}{ Sex } & Male & $111(69.4)$ & $148(68.8)$ & 0.911 \\
\hline & Female & 49 (30.6) & $67(31.2)$ & \\
\hline \multirow[t]{3}{*}{ Age (years) ${ }^{a}$} & $<60$ & $45(28.1)$ & $67(31.2)$ & 0.577 \\
\hline & $60-69$ & $57(35.6)$ & $81(37.7)$ & \\
\hline & $70-81$ & $58(36.3)$ & $67(31.2)$ & \\
\hline \multirow[t]{3}{*}{ Histologic type } & Differentiated & $62(38.8)$ & $74(34.4)$ & 0.345 \\
\hline & Undifferentiated & $98(61.3)$ & $139(64.7)$ & \\
\hline & Others & $0(0.0)$ & $2(0.9)$ & \\
\hline \multirow{3}{*}{$\begin{array}{l}\text { Cancer stage }{ }^{\mathrm{b}} \\
\text { (Japanese Classification) }\end{array}$} & $\|$ & $39(24.4)$ & $67(31.2)$ & 0.143 \\
\hline & $\| \mathrm{A}$ & $68(42.5)$ & $95(44.2)$ & \\
\hline & $\| I \mathrm{~B}$ & $53(33.1)$ & $53(24.7)$ & \\
\hline \multirow[t]{4}{*}{ Site of first relapse ${ }^{c}$} & Local & $11(6.9)$ & $17(7.9)$ & 0.707 \\
\hline & Lymph nodes & $30(18.8)$ & $53(24.7)$ & 0.173 \\
\hline & Peritoneum & $76(47.5)$ & $97(45.1)$ & 0.647 \\
\hline & Hematogenous & $59(36.9)$ & $69(32.1)$ & 0.334 \\
\hline \multicolumn{5}{|l|}{ Treatment after relapse } \\
\hline \multirow[t]{2}{*}{ Chemotherapy } & Yes & $121(75.6)$ & $171(79.5)$ & 0.367 \\
\hline & No & $39(24.4)$ & $44(20.5)$ & \\
\hline \multirow[t]{2}{*}{ Chemotherapy including S-1 } & Yes & 57 (35.6) & $132(61.4)$ & $<0.001$ \\
\hline & No & $103(64.4)$ & 83 (38.6) & \\
\hline \multirow[t]{2}{*}{ Surgery } & Yes & $13(8.1)$ & $23(10.7)$ & 0.403 \\
\hline & No & $147(91.9)$ & $192(89.3)$ & \\
\hline \multirow[t]{2}{*}{ Radiotherapy } & Yes & $5(3.1)$ & $4(1.9)$ & 0.429 \\
\hline & No & 155 (96.9) & $211(98.1)$ & \\
\hline
\end{tabular}

${ }^{\mathrm{a}}$ Age at time of recurrence

${ }^{\mathrm{b} C a n c e r ~ s t a g e: ~ J a p a n e s e ~ C l a s s i f i c a t i o n ~ o f ~ G a s t r i c ~ C a r c i n o m a ~(S e c o n d ~ E n g l i s h ~ E d i t i o n) ~}$

'Some patients had an initial recurrence at more than one site

group, $n=121$; surgery-only group, $n=171$ ), the median duration of follow-up after recurrence was 41.4 months. The median TFRD was 12.2 months (95\% CI, 8.6-15.0) in the adjuvant S-1 group and 12.7 months (95\% CI, 10.413.8) in the surgery-only group. The HR for death in the adjuvant S-1 group, as compared with the surgery-only group, was $1.12(95 \% \mathrm{CI}, 0.87-1.44)$ (Fig. 2b). The regimens used for the patients who received chemotherapy after recurrences are shown in Additional file 2: Figure S1a.

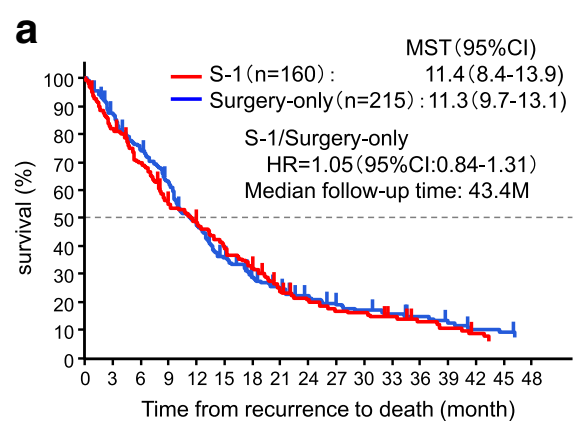

Fig. 2 TFRD in cohort 1. TFRD among (a) all patients who had recurrent disease and (b) patients who received chemotherapy after the development of recurrent disease

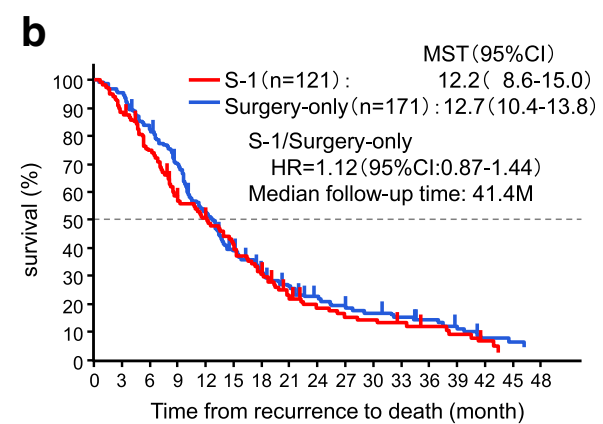


TFRD according to the chemotherapy regimen used after recurrence: IS vs. NIS

The details of the IS or NIS groups are shown in Table 2. In both treatment groups combined, the median duration of follow-up after recurrence was 41.4 months. The median TFRD was 13.9 months (95\% CI, 12.7-15.6) in the IS group $(n=189)$ and 8.1 months (95\% CI, 6.6-9.7) in the NIS group $(n=103)$. The HR for death in the IS group, as compared with the NIS group, was 0.59 (95\% CI, $0.45-0.76$ ) (Fig. 3a). In the adjuvant $\mathrm{S}-1$ group, the median duration of follow-up after recurrence was 41.4 months. The median TFRD was 14.9 months (95\% CI, 12.3-19.4) in the IS group $(n=57)$ and 8.2 months (95\% CI, 6.5-11.6) in the NIS group $(n=64)$. The HR for death in the IS group, as compared with the NIS group, was 0.61 (95\% CI, 0.42-0.91) (Fig. 3b). In the surgery-only group, the median duration of follow-up from recurrence was 38.6 months. The median TFRD was 13.8 months (95\% CI, 12.2-15.8) in the IS group $(n=132)$ and 6.9 months (95\% CI, 4.8-9.9) in the NIS group $(n=39)$. The HR for death in the IS group, as compared with the NIS group, was 0.54 (95\% CI, 0.37-0.79) (Fig. 3c). The TFRD was analyzed according to sex, age, histologic type, stage, timing of recurrence after surgery, and type of recurrence (Fig. 4). In subgroup analysis, there was no significant interaction between the adjuvant S-1 group and surgery-only group $(P=0.63$, Additional file 3 : Table S1). Moreover, the interactions between the IS and NIS groups were analyzed in both treatment groups combined, the adjuvant S-1 group and the surgery-only group. There was no significant interaction except for the age subgroup in the adjuvant $\mathrm{S}-1$ group $(P=0.0059$, Fig. 4$)$. The outcomes were better in the IS group except for the age subgroups, irrespective of whether patients received S-1 as postoperative adjuvant chemotherapy.

TFRD according to the interval between completion of adjuvant chemotherapy and recurrence in the adjuvant S-1 group

The median TFRD was 13.4 months in the IS group and 6.8 months in the NIS group (HR, 0.57; 95\% CI 0.30-1. 08, $P=0.078$ ) among patients with a recurrence-free interval (RFI) of less than 6 months. The median TFRD was 15.2 months in the IS group and 11.0 months in the NIS group (HR, 0.67; 95\% CI 0.40-1.13, $P=0.13$ ) among patients with an RFI of 6 months or longer. The 2 groups were not sufficiently large to allow definitive statistical conclusions, but the IS group seemed to have better OS after recurrence, irrespective of the interval between completion of adjuvant treatment and recurrence.

\section{Influence on survival after recurrence (multivariate analysis)}

Among the eligible subjects, 375 patients who had recurrent gastric cancer (adjuvant S-1 group, $n=160$; surgeryonly group, $n=215$ ) were included in multivariate Cox

Table 2 Patient characteristics in cohort 2

\begin{tabular}{|c|c|c|c|c|}
\hline Cohort 2 & & $\begin{array}{l}\text { IS group } \\
(n=189)\end{array}$ & $\begin{array}{l}\text { NIS group } \\
(n=103)\end{array}$ & $\begin{array}{l}X \text { square test } \\
p \text {-value }\end{array}$ \\
\hline \multirow[t]{2}{*}{ Sex } & Male & $134(70.9)$ & $72(69.9)$ & 0.858 \\
\hline & Female & $55(29.1)$ & $31(30.1)$ & \\
\hline \multirow[t]{3}{*}{ Age (years) ${ }^{a}$} & $<60$ & $60(31.8)$ & $38(36.8)$ & 0.199 \\
\hline & $60-69$ & $68(36.0)$ & $42(40.8)$ & \\
\hline & $70-81$ & $61(32.3)$ & $23(22.3)$ & \\
\hline \multirow[t]{3}{*}{ Histologic type } & Differentiated & $71(37.6)$ & $39(37.9)$ & 0.906 \\
\hline & Undifferentiated & $117(61.9)$ & $63(61.2)$ & \\
\hline & Others & $1(0.5)$ & $1(1.0)$ & \\
\hline \multirow{3}{*}{$\begin{array}{l}\text { Cancer stage } \\
\text { (Japanese Classification) }\end{array}$} & $\|$ & $64(33.9)$ & $25(24.3)$ & 0.226 \\
\hline & $\| \mathrm{A}$ & $73(38.6)$ & $44(42.7)$ & \\
\hline & IIIB & $52(27.5)$ & $34(33.0)$ & \\
\hline \multirow[t]{2}{*}{ Time from surgery to relapse (years) } & $<1 \mathrm{y}$ & $56(29.6)$ & $41(39.8)$ & 0.078 \\
\hline & $\geq 1 \mathrm{y}$ & $133(70.4)$ & $62(60.2)$ & \\
\hline \multirow[t]{4}{*}{ Site of first relapse ${ }^{c}$} & Local & $16(8.5)$ & $2(1.9)$ & 0.027 \\
\hline & Lymph nodes & $51(27.0)$ & $24(23.3)$ & 0.491 \\
\hline & Peritoneum & 79 (41.8) & $55(53.4)$ & 0.057 \\
\hline & Hematogenous & $63(33.3)$ & $36(35.0)$ & 0.780 \\
\hline
\end{tabular}

\footnotetext{
${ }^{a}$ Age at time of recurrence

${ }^{\mathrm{b}}$ Cancer stage: Japanese Classification of Gastric Carcinoma (Second English Edition)

'Some patients had initial recurrence at more than one site
} 
b

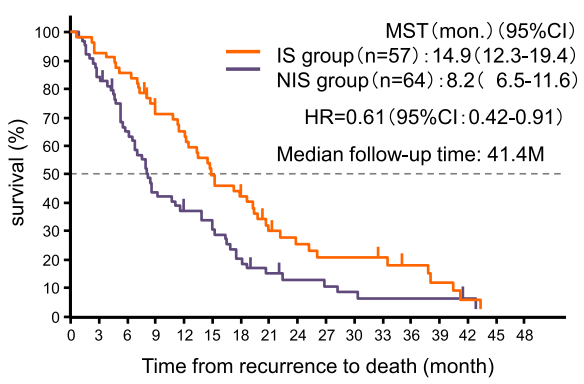

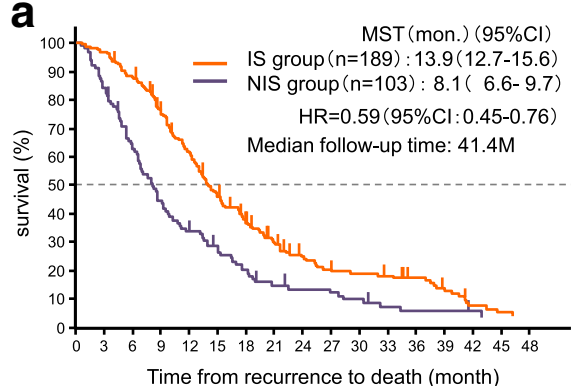

C

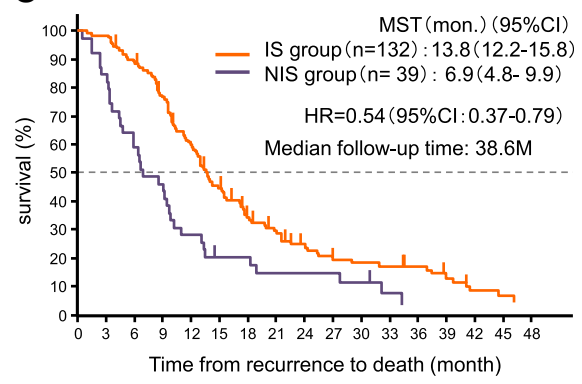

Fig. 3 TFRD in cohort 2. TFRD for patients who received regimens (a) including S-1 and those who received regimens not including S-1 after recurrence (both treatment groups combined), (b) including S-1 and those who received regimens not including S-1 after recurrence (adjuvant S-1 group) and (c) including S-1 and those who received regimens not including S-1 after recurrence (surgery-only group)

regression analysis to examine factors related to survival after recurrence (Additional file 4: Table S2). The HR for death was $0.64(95 \% \mathrm{CI}, 0.50-0.88, P<0.001)$ among 225 patients who had recurrence more than 1 year after surgery (after completion of adjuvant treatment), as compared with 120 patients who had recurrence within 1 year after surgery (within adjuvant treatment periods), suggesting that the interval from surgery to recurrence is a determinant of the duration of survival after recurrence. The regimens used for patients who received chemotherapy after recurrence are shown in Additional file 2: Figure S1b, S1c.

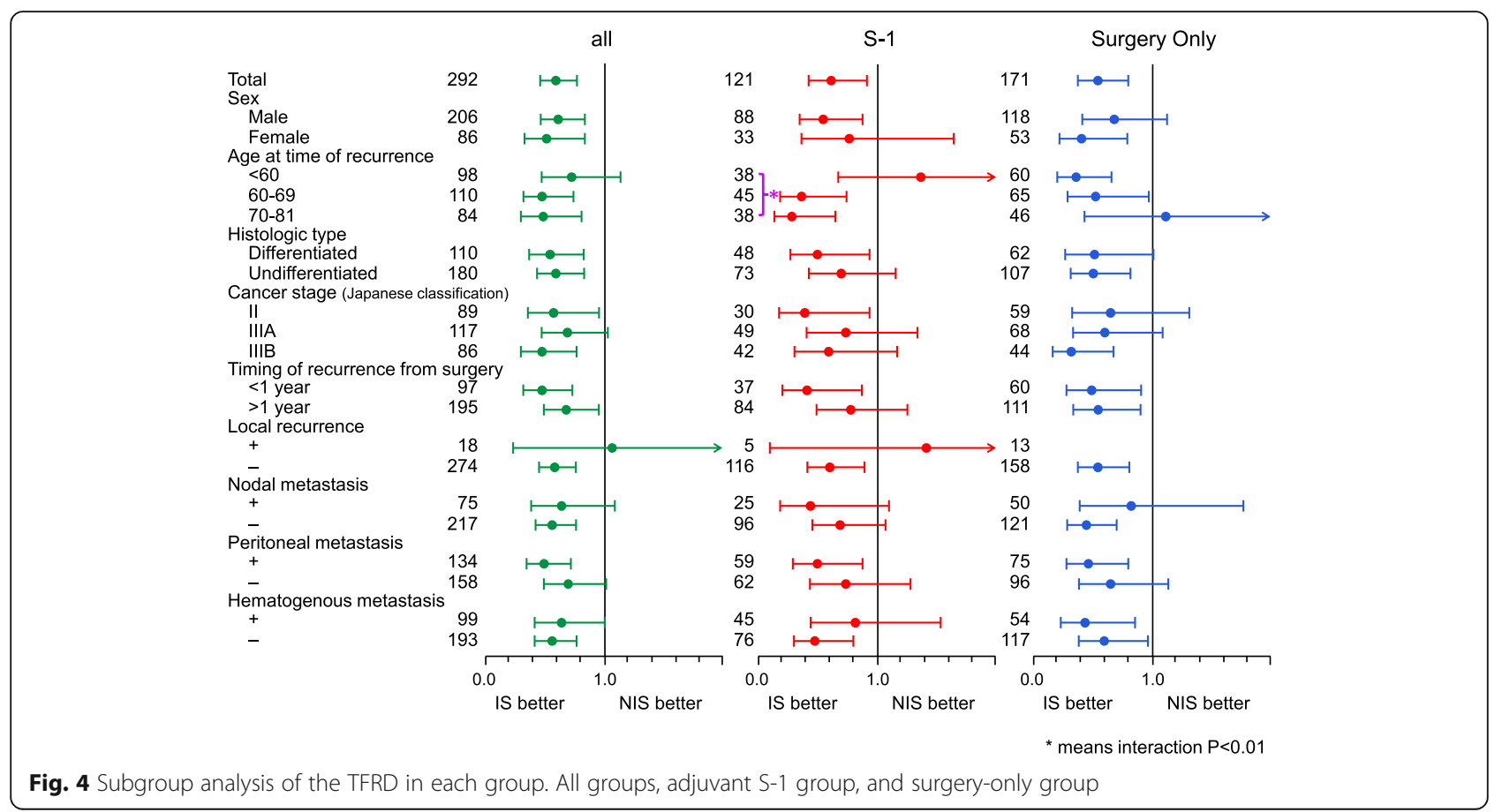




\section{Discussion}

Our study results suggest that the same treatment strategy can be applied to patients who develop recurrence after adjuvant chemotherapy and to those who have recurrence without adjuvant chemotherapy. To the best of our knowledge, similar studies, using results of phase III or meta-analysis, have not been performed in the field of gastric cancer. This analysis is the first investigation on this scale for gastric cancer. Two smaller retrospective reports [23, 24] have been previously published. Shitara et al. published a single-center and a multicenter retrospective study. In the single-center study, patients who developed recurrences after adjuvant chemotherapy with S-1 were retrospectively divided by whether they received S-1-containing regimen or non-S-1-containing regimen after recurrence. The non-S-1-containing regimen group had a high response rate and better progression-free survival than the $\mathrm{S}$-1-containing regimen group [23]. In contrast, the multicenter report showed that S-1 plus cisplatin (SP) therapy, as first-line chemotherapy after recurrence, yielded excellent results in patients with an RFI of at least 6 months [24].

In our exploratory analysis of data from the ACTSGC, patients who received adjuvant chemotherapy with S-1 tended to have a similar prognosis after recurrence as patients who did not receive adjuvant chemotherapy. In other words, prolonged OS in ACTS-GC study may directly reflect the relapse free survival (RFS) benefit of the adjuvant chemotherapy. This finding was consistent even when limiting the analysis to patients who received chemotherapy after recurrence.

Another clinical question is the selection of a regimen for patients with recurrence after adjuvant chemotherapy. Generally, as a principle of chemotherapy, reuse of the same drug is rarely considered for refractory patients. However, it is not clear whether the same strategy should be applied to patients with recurrence after adjuvant chemotherapy. Our analysis revealed that reuse of an S-1 including regimen after recurrence showed better results than a regimen without $\mathrm{S}-1$ for all populations (both treatment groups combined, adjuvant S-1 group, and surgery-only group).

There were some limitations to our analysis; for example, some of the detailed information on patient characteristics such as performance status at recurrence and availability of oral administration was lacking, and thus, the effects of such factors could not be excluded. To minimize any bias, subgroup analysis was performed according to factors that may influence prognosis, including age at recurrence, timing of recurrence, and site of recurrence. We found the IS regimen had a better outcome than NIS for almost all subgroups. An interaction was found only in the subgroup of age at recurrence in the adjuvant S-1 group, although the difference was not significant $(\mathrm{HR}=1.37,95 \% \mathrm{CI} 0.67-2.78)$. Thus, for patients younger than 60 years at the time of recurrence, a regimen not including $\mathrm{S}-1$ should be considered.

The XParTS trial, a phase II trial with a small sample size, investigated the efficacy of reuse of fluoropyrimidinebased anticancer agents in patients receiving adjuvant chemotherapy with $\mathrm{S}-1$, and its results suggested this regimen's efficacy in patients with recurrence after adjuvant chemotherapy [25]. This finding supports our analysis results. Currently, a phase II trial (KSCC1001, UMIN ID: UMIN000004303) is investigating the efficacy of SP therapy in patients with gastric cancer who had recurrence at least 6 months after adjuvant chemotherapy with S-1, and a randomized phase II trial (OGSG1103, UMIN ID: UMIN000006105) is investigating the efficacy of SP and capecitabine plus cisplatin therapies in patients with gastric cancer who had recurrences after adjuvant chemotherapy with S-1. We may need to re-interpret our analysis results in the context of the results of these studies.

\section{Conclusions}

Our analysis showed that postoperative adjuvant chemotherapy with S-1 for stage II or III gastric cancer prolonged recurrence-free survival without influencing the TFRD. Furthermore, our results suggest that S-1-based regimens may be effective for the management of patients with recurrent gastric cancer who received S-1 as adjuvant chemotherapy. Patients with recurrence after adjuvant chemotherapy with S-1 may have the same degree of sensitivity to chemotherapy for recurrent gastric cancer as those who develop recurrences without adjuvant chemotherapy. Thus, the same treatment strategy may be applied to patients who develop recurrences after adjuvant chemotherapy and those who did not receive adjuvant chemotherapy.

\section{Additional files}

Additional file 1: Text S1. Institutions participating in the ACTS-GC.
(DOCX $30 \mathrm{~kb})$

Additional file 2: Figure S1. Chemotherapeutic regimens used after recurrence. Chemotherapeutic regimens used after recurrence in (a) all recurrence patients, (b) patients who had recurrence within 1 year after surgery, and (c) patients who had recurrence more than 1 year after surgery. (DOCX $166 \mathrm{~kb}$ )

Additional file 3: Table S1. Subgroup analysis of the time from recurrence to death in each group (detail for Fig. 4). (DOCX 51 kb)

Additional file 4: Table S2. Multivariate Cox regression analysis of survival after recurrence. (DOCX 29 kb)

\section{Abbreviations}

ACTS-GC: Adjuvant Chemotherapy Trial of S-1 for Gastric cancer; BSA: Body surface area; $\mathrm{Cl}$ : Confidence interval; HR: Hazard ratio; IS: Including S-1; NIS: Not including S-1; OS: Overall survival; RFI: Recurrence-free interval; TFRD: Time from recurrence to death 


\section{Acknowledgements}

We thank all of the patients enrolled in the ACTS-GC and their families.

\section{Funding}

This study was sponsored by Taiho Pharmaceutical Co., LTD. Taiho Pharmaceutical Co., LTD funded and was involved in the study design, data collection, data analysis, data interpretation, writing support of this report, and reviewed the manuscript before the submission.

\section{Availability of data and materials}

The datasets generated and analyzed during this analysis are not publicly available, due to language concerns and patient privacy, but are available from the corresponding author on reasonable request.

\section{Authors' contributions}

SI and MS contributed to the conception and design of this analysis. YO was responsible for the statistical analysis. SI and YO had full access to all of the data in the study and take responsibility for the integrity and accuracy of the data analysis. All authors contributed to the acquisition, analysis, or interpretation of data. SI drafted the manuscript. All authors contributed to the critical revision of the manuscript for important intellectual content. All authors had final responsibility for the decision to submit the manuscript for publication. In addition, all authors read and approved the final manuscript.

\section{Ethics approval and consent to participate}

The ACTS-GC trial was conducted in accordance with the World Medical Association Declaration of Helsinki and Japanese Good Clinical Practice guidelines. The protocol of ACTS-GC was approved by the institutional review board of each participating hospital. The institutions participated in the ACTS-GC were shown in Additional file 1: Text S1. Written informed consent was obtained from all patients. Since this analysis was an ad-hoc exploratory analysis, we did not seek additional approval and consent.

\section{Competing interests}

SI received honoraria from Taiho, Chugai, Yakult Honsha, Covidien, Eli Lilly, Takeda, Nippon Kayaku, Kyowa Hakko Kirin, and a grant from Chugai, Yakult Honsha, and writing assistance from Taiho; YO has stock in Statcom and received personal fees as executive salaries from Statcom, honoraria from Sanofi and Eisai, and consulting fees from Chugai, Taiho, Shionogi, Kowa and Eisai, and travel expenses from Yakult Honsha, and Takeda; MS received honoraria from Taiho, Yakult Honsha, Chugai, Eli Lilly, Ono, Takeda, and Sanofi, and grants from Taiho, Yakult Honsha, Eli Lilly, Sanofi, and Takeda.

\section{Publisher's Note}

Springer Nature remains neutral with regard to jurisdictional claims in published maps and institutional affiliations.

\section{Author details}

'Department of Gastroenterological Surgery, Aichi Cancer Center, 1-1 Kanokoden, Chikusa-ku, Nagoya, Aichi 464-8681, Japan. ${ }^{2}$ Department of Integrated Science and Engineering for Sustainable Society, Chuo University, 1-13-27 Kasuga, Bunkyo-ku, Tokyo 112-8551, Japan. ${ }^{3}$ Department of Surgery, Hyogo College of Medicine, 1-1 Mukogawa-cho, Nishinomiya City, Hyogo 663-8501, Japan.

\section{Received: 16 April 2017 Accepted: 8 April 2018}

\section{Published online: 20 April 2018}

\section{References}

1. Ferlay J, Soerjomataram I, Dikshit R, Ester S, Mathers C, Rebelo M, et al. Cancer incidence and mortality worldwide: sources, methods and major patterns in GLOBOCAN 2012. Int J Cancer. 2015;136(5):E359-86.

2. Earle CC, Maroun JA. Adjuvant chemotherapy after curative resection for gastric cancer in non-Asian patients: revisiting a meta-analysis of randomised trials. Eur J Cancer. 1999;35(7):1059-64.

3. Hermans J, Bonenkamp JJ, Boon MC, Bunt AM, Ohyama S, Sasako M, et al. Adjuvant therapy after curative resection for gastric cancer: meta-analysis of randomized trials. J Clin Oncol. 1993;11(8):1441-7.

4. Mari E, Floriani I, Tinazzi A, Buda A, Belfiglio M, Valentini $M$, et al. Efficacy of adjuvant chemotherapy after curative resection for gastric cancer: a meta-analysis of published randomised trials. A study of the
GISCAD (Gruppo Italiano per lo studio dei Carcinomi dell'Apparato Digerente). Ann Oncol. 2000;11(7):837-43.

5. Panzini I, Gianni L, Fattori PP, Tassinari D, Imola M, Fabbri P, et al. Adjuvant chemotherapy in gastric cancer: a meta-analysis of randomized trials and a comparison with previous meta-analyses. Tumori. 2002:88(1):21-7.

6. GASTRIC (Global Advanced/Adjuvant Stomach Tumor Research International Collaboration) Group, Paoletti X, Oba K, Burzykowski T, Michiels S, Ohashi Y, et al. Benefit of adjuvant chemotherapy for resectable gastric cancer: a meta-analysis. JAMA. 2010;303(17):1729-37.

7. Piedbois P, Buyse M. Meta-analyses need time, collaboration, and funding. J Clin Oncol. 1994;12(4):878-80.

8. Bang YJ, Kim YW, Yang HK, Chung HC, Park YK, Lee KH, et al. Adjuvant capecitabine and oxaliplatin for gastric cancer after D2 gastrectomy (CLASSIC): a phase 3 open-label, randomised controlled trial. Lancet. 2012; 379(9813):315-21.

9. Noh SH, Park SR, Yang HK, Chung HC, Chung IJ, Kim SW, et al. Adjuvant capecitabine plus oxaliplatin for gastric cancer after D2 gastrectomy (CLASSIC): 5-year follow-up of an open-label, randomised phase 3 trial. Lancet Oncol. 2014;15(12):1389-96.

10. Japanese Gastric Cancer Association. Japanese classification of gastric carcinoma-2nd English edition. Gastric Cancer. 1998;1:10-24.

11. Shirasaka T, Shimamato Y, Ohshimo H, Yamaguchi M, Kato T, Yonekura K, et al. Development of a novel form of an oral 5 -fl uorouracil derivative (S-1) directed to the potentiation of the tumour selective cytotoxicity of 5-fluorouracil by two biochemical modulators. Anti-Cancer Drugs. 1996;7(5): $548-57$.

12. Sakuramoto S, Sasako M, Yamaguchi T, Kinoshita T, Fujii M, Nashimoto A, et al. Adjuvant chemotherapy for gastric cancer with S-1, an oral fluoropyrimidine. N Engl J Med. 2007;357(18):1810-20.

13. Sasako M, Sakuramoto S, Katai H, Kinoshita T, Furukawa H, Yamaguchi T, et al. Five-year outcomes of a randomized phase iii trial comparing adjuvant chemotherapy with S-1 versus surgery alone in stage ii or iii gastric cancer. J Clin Oncol. 2011;29(33):4387-93.

14. O'Connell MJ, Campbell ME, Goldberg RM, Grothey A, Seitz JF, Benedetti JK, et al. Survival following recurrence in stage II and III colon cancer: findings from the ACCENT data set. J Clin Oncol. 2008;26(14):2336-41.

15. André T, Boni $C$, Navarro M, Tabernero J, Hickish T, Topham C, et al. Improved overall survival with oxaliplatin, fluorouracil, and leucovorin as adjuvant treatment in stage II or III colon cancer in the MOSAIC trial. J Clin Oncol. 2009;27(19):3109-16.

16. Schmol HJ, Twelves C, O'Connel MJ, O'Connell MJ, Cartwright T, McKenna E, et al. Effect of adjuvant capecitabine or fluorouracil, with or without oxaliplatin, on survival outcomes in stage III colon cancer and the effect of oxaliplatin on post-relapse survival: a pooled analysis of individual patient data from four randomised controlled trials. Lancet Oncol. 2014;15(13): $1481-92$.

17. Kuebler JP, Wieand HS, O'Connell MJ, Smith RE, Colangelo LH, Yothers G, et al. Oxaliplatin combined with weekly bolus fluorouracil and leucovorin as surgical adjuvant chemotherapy for stage II and III colon cancer: results from NSABP C-07. J Clin Oncol. 2007;25(16):2198-204.

18. Markman M, Rothman R, Hakes T, Reichman B, Hoskins W, Rubin S, et al. Second-line platinum therapy in patients with ovarian cancer previously treated with cisplatin. J Clin Oncol. 1991;9(3):389-93.

19. Parmar MK, Ledermann JA, Colombo N, du Bois A, Delaloye JF, Kristensen $G B$, et al. Paclitaxel plus platinum-based chemotherapy versus conventional platinum-based chemotherapy in women with relapsed ovarian cancer: the ICON4/AGO-OVAR-2.2 trial. Lancet. 2003;361(9375):2099-106.

20. Harries M, Gore M. Part II: chemotherapy for epithelial ovarian cancer-treatment of recurrent disease. Lancet Oncol. 2002;3(9):537-45.

21. Fung-Kee-Fung M, Oliver T, Elit L, Oza A, Hirte HW, Bryson P. Optimal chemotherapy treatment for women with recurrent ovarian cancer. Curr Oncol. 2007;14(5):195-208.

22. Lang I, Bell R, Feng FY, Lopez Rl, Jassem J, Semiglazov V, et al. Trastuzumab retreatment after relapse on adjuvant trastuzumab therapy for human epidermal growth factor receptor 2-positive breast cancer: final results of the retreatment after HErceptin adjuvant trial. Clin Oncol (R Coll Radiol). 2014;26(2):81-9.

23. Shitara K, Muro K, Ura T, Takahari D, Yokota T, Sawaki A, et al. Chemotherapy for gastric cancer that recurs after adjuvant chemotherapy with S-1. Jpn J Clin Oncol. 2008;38(11):786-9. 
24. Shitara K, Morita S, Fujitani K, Kadowaki S, Takiguchi N, Hirabayashi N, et al Combination chemotherapy with S-1 plus cisplatin for gastric cancer that recurs after adjuvant chemotherapy with S-1: multi-institutional retrospective analysis. Gastric Cancer. 2012;15(3):245-51.

25. Nishikawa K, Tsuburaya A, Yoshikawa T, Takahashi M, Tanabe K, Yamaguchi K, et al. A phase II trial of capecitabine plus cisplatin (XP) for patients with advanced gastric cancer with early relapse after S-1 adjuvant therapy: XParTS-I trial. Gastric Cancer. 2018. Epub ahead of print.

Ready to submit your research? Choose BMC and benefit from:

- fast, convenient online submission

- thorough peer review by experienced researchers in your field

- rapid publication on acceptance

- support for research data, including large and complex data types

- gold Open Access which fosters wider collaboration and increased citations

- maximum visibility for your research: over $100 \mathrm{M}$ website views per year 